A. W. Wolshenski, Ju. Ss. Burow, B. N. Winogradow und K. W. Gladkich, Betone und Erzeugnisse aus Schlakken- und Aschezementen. (Bei Härtung in Dampfkammern und Autoklaven). Moskau: Gosstroisdat. 1963. (362 S. m. Abb.) 1 Rbl. 32 Kop. [russ.]

$\mathrm{H}_{8}$. Metallurgie, Korrosion

R. Kieffer und F. Benesovsky, Hartstoffe. Wien: SpringerVerl. 1963. (XII + 602 S. m. 179 Abb.) 80. DM 114,-

Johannes Reiprich, Aluminium-Taschenbuch. 12., neubcarb. u. wesentl. erw. Aufl. Herausgeg, von der Aluminium-Zentrale, Düsscldorf. Düsseldorf: Alnminium-Verl. 1963. (XVIII + $\$ 32$ S. m. 384 Abb. u. 176 Taf.) $8^{\circ}$. DM 29,60 .

Hermann Schumann, Metallographic, 4., verb. Aufl Leipzig: Deutscher Verl. für Grundstoffindustrie. 1962. (611 S. m. 1012 Abb. u. 4 Taf.) DM 38,--.

-, Gußeisen. Handbuch für Gußerzeuger und Gußverbraucher. Aus dem Russischen übersetzt von Karl Uebrick. Leipzig: Deutscher Verl. für Grundstoffindustrie. 1963. (426 S. m. $344 \mathrm{Abb}$. u. $254 \mathrm{Tab}$.) $8^{0}$ DM 46,- .

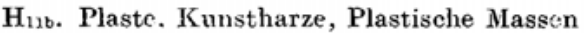

Waldemar Beyer, Glasfaserverstärkte Kunststoffe. 3., erg. u. erw. Aufl. München: Carl Hanser Veri. 1963. $(129 \mathrm{~S} . \mathrm{m} .82 \mathrm{Abb}$, u. $10 \mathrm{Taf})=$. Kunststoff-Ver arbeitung. Folge 2. DM 9,80 .

Carlowitz, Kunststoff-Tabellen. Teil I-XIV. BensbergFrankenforst: Fritz Schiffmann. 1963. (248 S.) DM 19,80 .

Georg Haim, 25 Jahre Kunststoff-Schweißen. München: Carl Hanser Verl. 1963. (95 S. m. 52 Abb.) DM 12,80.

C. M. v. Meysenbug, Kunststoffkunde für Ingenieure. Das Fachwissen des Ingenieurs. München: Carl Hanser Verl. 1963. (188 S. m. 125 Abb. u. 20 Taf.) DM 19,80.

A. Rost, Verarbeitungstechnik der Epoxyd-Gießharze. München: Carl Hanser Verl. 1963. (132 S. m. $90 \mathrm{Abb}$. u. 10 Taf. $=$ Kunststoff-Verarbeitung. Folge 9 DM 17,80 .

Irene Saechtling, Kunststoff-Lieferliste für Werkstatt und Betrieb. München: Carl Hanser Verl. 1963. (52 S.) DM 4,50.

Hansjürgen Saechtling, Die Herkunft der Kunststoffe. 3. völlig neubearb. Aufl. München: Carl Hanser Verl. 1963. (4 Taf. m. Erläuterungen u. Register) DII 6,80.

$\mathrm{H}_{16}$. Nahrungs., Genuß- und Futtermittel

N. I. Tkatschew, Plastische Massen und ihre Anwendung in der Back. und Hefeindustrie. Moskau: Pisch tschepromisdat. 1963. (224 S. m. Abb.) 79 Kop. [russ.]

$\mathrm{H}_{17}$. Fette, Seifen, Wasch- und Reinigungsmittel, Wachse, Bohncrmassen usw.

H. P. Kaufmann, Neuzeitliche Technologie der Fette und Fettprodnkte. 3. Lfg.: Die (Aewinnung der Öle und Fette aus den Rohstoffen: Grundlagen und Pressung. Münster: Aschenidorffsche Verlagsbuchhandlung. 1961 $(1 V+167$ S. m. Abb. u. Tab.) DM 25.-

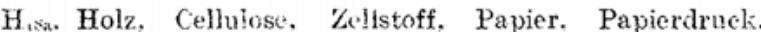
Celluloid, Linoieun

Dietrich Hoyer, Handbuch der Kartor- und Pappen herstellung. 1. Aufi. Leipzig: Fachbuchveri. 1963. (325 S. m. 200 A bb. u. 44 T'ab.) DM 21.-.

$\mathrm{H}_{\mathrm{i} 8 \mathrm{~b}}$. Textilfasern.

Radko Krěma u. a., Textilverbundstoffe. Ubersetzt aus dem Tschechischen. Leipzig: Fachbuchver!. 1963. (260 S. m. Abb. u. Tab.) DM 18,20.
$\mathrm{H}_{20}$. Schieß- und Sprengstoffe, Zündmittel

Karlheinz Lohs, Nachweisgeräte für giftige Gase, Dämpfe und Staube. 2., überarb. u. erg. Aufl. Berlin: Deutscher Militärverl. 1962. (213 S.) DM 4,20.

Ralf Stöhr, Die chemischen Kampfstoffe. Eigenschaften, Wirkung, Schutzmöglichkeiten und Entgiftung. Ber lin: Deutscher Militärverl. 1961. (227 S.) DM 6,50.

$\mathrm{H}_{21}$. Leder, Gerbstoffe

B. Ja. Kipniss, W. N. Kolessnikow, D. W. Lerner u. a. Hrsg., Handbuch über die Herstellung von Kunst. leder (in 2 Bd.). Bd. 1. Moskau: Gislegprom. 1963. (524 S.) 1 Rbl. 83 Kop. [russ.]

-, Chemisch-technische Untersuchungsmethoden für die Lederindustrie. 2. Aufl. Herausgeg. von E. Merck. Weinheim/Bergstr.: Verl. Chemie. 1958. (XVI + $238 \mathrm{~S}$. m. 18 Abb.) 80. DM 16.50.

$\mathrm{H}_{24}$. Photographie

Gerhard Teicher, Hrsg., Handbuch der Fototechnik. Halle: Fotokinovarl. 1963. (893 S. m. $796 \mathrm{Abb}, \mathrm{u}$. 94 Tab.) DM $59,-$.

Anmerkung!

Die Bibliographienbeilage: erscheinen $\mathbf{z w a n g l o s}$ und fort laufend numeriert. Die Seiten der Beilagen sind mit B ge. kemmeichnet und werden durchpaginiert. Die Bibliographien werden für das Autorenregister und Sachregister ausgewertet.

\section{Ausgewertete Biblingraphie-Fachzeitschriften}

Beigien Bibliographie de Belgique - Belgische Biblio. graphie

ĆSSR Bibliography of Publications Dealing with the Polarography method [Praha]

Bulletin - Die Neuesten Forschungserge b. nisse der Tschechoslowakischen Wissenschaft Nové Knihy (Neve Bücher)

Novinky (Neuheiten)

Däncmark Danske Bogmarked

Deutschland Börsenblatt für den Deutschen Buchhandel - Frankfurter Ausgabe Deutsche Bibliographie. Wöchentliches Verzeichis. Ausgabe II. [Frankfurt/Main] Deutsche Nationalbibliographie. Reihe A Neuerscheinungen des Buchhandels [Leipzig]

Englard British National Bibliograply

Frankreich 13ibliographie de la France

Griechenland Greet Bibliography [Athen]

Italien Bibliografin Nazional: Italiana

Nickerlande Niruswblad voor de Boekhandel

Österreich Östraruichisehe Bibliographie

Schweiz Schweizer Buch. Seris A und Serie B

UdSSR Rücherverzichnis des Jahres Neue Bücher

CSA Publishers' Weekly 